\title{
La estructura analógica de la «quinta vía» de Santo Tomás de Aquino
}

\author{
The analogical structure of the "Fifth Way» \\ of Saint Thomas Aquinas
}

\author{
DESIDERIO PARRILLA MARTÍNEZ \\ Universidad católica de Murcia (UCAM)
}

Recibido: 30/11/16 Aceptado: 22/12/16

\section{RESUMEN}

Tanto en la Suma contra gentiles como en la Suma teológica la quinta vía posee la estructura de una analogía de proporcionalidad. No obstante, cada una de las Sumas utiliza de manera diferente esta idea de Dios como «inteligencia ordenadora» obtenida por analogía. En Suma contra gentiles se usa este término análogo en un plan de disputa dialéctica, mientras que en Suma Teológica se aplica dicho término a una analogía de atribución para explicar la acción creadora. El artículo explica las razones de esta doble estrategia desarrollada, sin embargo, a partir de una misma analogía inicial.

PALABRAS CLAVE: TOMÁS DE AQUINO, TELEOLOGÍA, EXISTENCIA DE DIOS, ANALOGÍA.

\section{ABSTRACT}

Such in Summa contra Gentiles as in Summa Theologica the «fifth way» has the structure of an analogy of proportionality. However, each of the Summas used differently this idea of God as «intelligent designer» obtained by analogy. In Summa contra Gentiles this analogue is used in a dialectical plan, while in Summa Theologica that term to an analogy of attribution is applied to explain the creative action. The article explains the reasons for this double strategy developed, however, from the same initial analogy.

\section{KEYWORDS:}

THOMAS AQUINAS, TELEOLOGY, EXISTENCE OF GOD, ANALOGY. 


\section{CONTEXTO HISTÓRICO.}

La «quinta vía» se incluye en la primera parte de la Suma Teológica (ST), escrita durante la estancia como maestro regente en Roma, desde 1265 hasta septiembre de 1268. La vía parece, sin embargo, ya establecida en el libro primero de la Suma contra gentiles (SCG) cuyos primeros 53 capítulos se redactan en París antes del verano de 1259, y se revisan en Italia durante 1260.

Mandonnet atribuye el Comentario de los ocho libros de los Físicos a esta misma estancia, en 1265, antes de la Prima pars. Es cierto que pocos han mantenido esta tesis, de la que dudan otros autores reputados (Grabmann, Castagnoli, Mansion) y Eschmann proponía con ellos situar este comentario en París entre 1268 y 1271. Weisheipl propone incluso una fecha más tardía: 1271. Gauthier data su redacción en el curso escolar 1268-1269, en el segundo período de enseñanza en París. Sin embargo, respecto a la cronología de las obras asumimos el canon consolidado con Jean-Pierre Torrell y Gilles Emery como superación de planteamientos completamente descartados. ${ }^{1}$

Por tanto, sin entrar en cuestiones de detalle, preterimos la cuestión de la redacción material del comentario, pero asumimos como probable la datación de Mandonnet solamente en lo tocante a la lectura exhaustiva, y estudio detenido, que desembocará en su composición final. Lo hacemos motivados sobre todo por el radical cambio de planteamiento que la vía sufre en ST (1265) respecto a su exposición previa en la SCG (1261) y que tiene el mencionado comentario como revulsivo.

Ciertamente, en SCG se alude explícitamente al capítulo 8 del segundo libro de Física, pero en ST la vía queda considerablemente transformada desde las coordenadas de este pasaje aristotélico, aunque no se cite en autoridades. A nuestro modo de ver una re-exposición tan severa de la «quinta vía» queda sin explicar si no se admite que la Prima pars se dicta mientras acomete el comentario de Física II 8, bien como lector bien en su fase de redacción más primaria. Además, es notorio que en su Comentario a la Física (In Physic. 11, lect. 12, n. 250) se contiene un germen de la fórmula definitiva de la vía ${ }^{2}$ lo suficientemente desarrollado como para ser indicio de esta contigüidad entre ambas fuentes.

Por lo demás, la importancia decisiva de Física II 8 se pone de relieve desde las formulaciones más primitivas de la vía. El libro primero del Comentario a las Sentencias de Pedro Lombardo enuncia, siguiendo a Dioniso el Aeropagi-

1 Jean-Pierre Torrell, Iniciación a Tomás de Aquino: su persona y su obra, Pamplona, EUNSA, 2002, pp. 483-525, 611-632.

2 «Natura est de numero illarum causarum quae propter aliquid agunt. Et hoc valet ad quaestionem de providentia. Ea enim quae non cognoscunt finem, non tendunt in finem nisi ut directa ab aliquo cognoscente, sicut sagitta a sagittante; unde si natura operatur propter finem, necesse est quod ab aliquo intelligente ordinetur; quod est providentiae opus». 
ta, una vía según la causalidad final para demostrar la existencia de Dios «ex gubernatione rerum». ${ }^{3}$ Esta vía establece precisamente el esquema de la vía en ST, mientras que el libro segundo del Comentario contiene la misma prueba enunciada «ex ipso ordine universi» ${ }^{4}$ según el esquema que encontraremos reproducido en SCG.

La cuestión disputada De potentia data muy probablemente del primer año de este período (1265-66), antes de la redacción de la Prima pars. El estilo de la «quinta vía» formulada en esta cuestión disputada manifiesta, por contraste, el quiebro que supone la «vía finalista $»^{5}$ en la ST, estableciendo un antes y un después en su formulación. Aunque las cuestiones disputadas De veritate datan de los tres años de enseñanza magistral de Tomás en París, desde 1256 hasta 1259, sin embargo también se disputó por extenso la «vía teleológica» en las líneas directrices que la SCG retoma, ${ }^{6}$ al menos durante el primer año de regencia.

Como trataremos de argumentar a continuación, el Tratado De Deo Uno y la propia «quinta vía», constituyen los textos más aristotélicos del corpus tomístico. Ya en la Prima secundae (1271) se pierde este tono aristotélico, que se recrudece en textos posteriores, como la exposición de la «vía teleológica» en el Prólogo al Comentario al evangelio de San Juan (1270-72) y que se radicaliza en la collatio del artículo 1 del Comentario al Símbolo de los Apóstoles (1273), contemporáneos por lo demás del Comentario a la Metafísica donde expone de nuevo la «vía teleológica» de forma implícita. ${ }^{7}$

Un cierto número de especialistas modernos ha advertido las visibles diferencias entre la primera y la segunda parte de la ST: ${ }^{8}$ «Eschmann constata el hecho al examinar el tratamiento de algunas tesis desde los comienzos de Prima secundae en adelante. El tono de la Prima pars es fríamente metafísico, preciso y abrupto, incluso al hablar de la providencia divina. Los teólogos interpretan este contraste por la diferente materia tratada en las dos partes. Sin embargo, el problema parece ser más profundo, porque -como vemos- afecta a todos sus escritos posteriores. Gauthier mantiene que Tomás fue inducido a «mitigar el intelectualismo excesivo que había desplegado anteriormente». Don Otón Lottin achaca este viraje a la condena del 10 de diciembre de 1270 del cardenal Tempier contra el averroísmo latino. Santiago Ramírez lo achaca a una creciente influencia de San Agustín en la obra de Tomás».

3 In I Sent., d. 35, q. 1, a. 1.

4 In II Sent., d. 1, q. 1, a. 1.

5 De potentia, q. 3 , a. 6, c.; q. 3 a. 15 co.

6 De veritate, q. 5 a. 1-15.

7 Comentario a la Metafísica de Aristóteles, libro XII, capítulo 10, lectio 12.

8 Cfr. James A. Weisheipl, Tomás de Aquino. Vida, obra y doctrina, Navarra, EUNSA, 1994, pp. 284-285. 
Con el presente artículo proponemos que este cambio no obedece principalmente a motivos extrínsecos (una cautela ante la condena de las tesis averroístas, por ejemplo) sino a razones de orden interno, entre las que destacamos su conciliación de la doctrina aristotélica establecida en Física II 8 con la doctrina de la creación y la providencia divina, que tiene la «quinta vía» como experimentum crucis.

\section{LA «QUINTA VÍA» EN LA SUMA CONTRA GENTILES.}

La primera formulación de la vía en SCG aparece en el libro I capítulo 13, donde realiza una formulación tentativa que recoge algunos antecedentes de la tradición. Esta formulación inicial asimila un éndoxon heredado directamente de San Juan Damasceno y de Averroes ${ }^{9}$ sin que falte la pertinente cita, aunque no desarrollo, de la Física de Aristóteles (libro II capítulo 8). A medida que Tomás despliega el plan de la Suma perfecciona dicha vía inicial conforme a tres ejes de desarrollo evolutivo:

a) El conocimiento de Dios se obtiene por una analogía de proporcionalidad propia a partir de los seres del mundo. Las referencias al respecto son tan abundantes como dispersas y no hacen sino reiterar el mismo asunto sucesivamente: lib. 1 cap. 34 n. 1 , lib. 2 cap. 37 n. 3 , lib. 3 cap. 54 n. 14;

b) en paralelo, dicha analogía se aplica a la proporción entre los seres naturales y los seres artificiales. ${ }^{10}$ Nos atrevemos a dar en batería algunas evidencias textuales, de entre todas las posibles, para corroborar este quiasmo lógico: lib. 1 cap. 66 n. 3, lib. 2 cap. 26 n. 6 . En algún fragmento establece directamente la analogía entre la finalidad de los seres naturales en comparación con los seres artificiales, como en lib. 3 cap. 2 n. 7. En definitiva, expone la analogía germinal que Aristóteles formula en Física II 8 y que se convierte en el locus clásico del axioma: «la obra de naturaleza es obra de inteligencia».11

9 Quizás este capítulo 13 sea uno de los que mayor número de comentarios ha suscitado en torno a los escritos de Santo Tomás. Remitimos a artículos clásicos donde se encuentra la mejor bibliografía clásica al respecto. Bulletin thomiste 10 (1933) n. 815-844, pp. 725-731; 4 (1935) n. 930-942, pp. 600-611; Archive d’histoire littéraire du Moyen Age 9 (1934) pp. 143-153. Como obra clásica para conocer las fuentes tomistas, ver: R. Arnou, S. J., De quinque viis S. Thomae ad demonstrandum Dei existentiam apud antiquos graecos et arabes et iudaeos praeformatis vel adumbratis textus selecti, Romae, Universitatis Gregorianae, 1932. In- $8^{\circ}$ de 104 p.

10 Contra Gentiles, lib. 3 cap. 100 n. 6.

11 Weisheipl, James A., «The Axiom, 'Opus naturae est opus intelligentiae' and Its Origins», en: Albertus Magnus-Doctor Universalis 1280-1980, G. Meyer and A. Zimmerman (ed.), Mainz: Matthias Grünewald-Verlag, 1980, pp. 446-447. Hödl, Ludwig, «Opus naturae est opus intelligentiae. Ein neuplatonisches Axiom im aristotelischen Verstàndnis des Albertus Magnus», en: Averroismus im Mittelalter und in der Renaissance, Friedrich Niewõhner, Loris Sturlese (eds.), 1994, pp. 132-148. Solmsen, Friedrich, «Nature as Craftsman in Greek Thought», 
Y finalmente c) la mencionada analogía entre lo natural y lo artificial presupone dos tipos de operaciones que se proporcionan entre Dios y el hombre: las acciones inmanentes y las acciones transeúntes. La analogía se traslada a su vez al paralelo entre la creación divina y las operaciones inmanentes y transeúntes, propias de las ciencias especulativas y las artes mecánicas respectivamente. ${ }^{12}$ De hecho, toda referencia al componente ontológico de la analogía se compone habitualmente con este segundo referente de cariz estrictamente gnoseológico.

A partir de esta endíadis de términos análogos queda establecido el esquema lógico básico para reconstruir la «quinta vía» en sus formas más complejas, en el marco más amplio de una doctrina de la creación. Estos tres ejes coordinados entre sí permiten considerar dicha creación como obra realizada con inteligencia y voluntad (acciones inmanentes) ${ }^{13}$ y como producto de la acción productora de Dios (acción transeúnte). ${ }^{14}$ Esta analogía de proporcionalidad entre la acción artesanal humana y la acción creadora divina es, en puridad, el núcleo de la estructura lógica de la «quinta vía». ${ }^{15}$ Tanto es así que el texto de la «quinta vía» de la ST en la edición tauritense de la Marietti remite directamente con una cita cruzada al corazón mismo de esta distinción en SCG lib. 2, cap. 24, que introduce las nociones de «producción» y de «operación transeúnte» como modelo analógico para explicar la acción creadora. Esta trenza lógica entre lo natural y lo artificial con las acciones transitivas e inmanentes se manifiesta particularmente obvia en la estructura simétrica de las notas 5 y 6 del capítulo 23, libro segundo, de Suma contra gentiles, donde se da in nuce la fórmula definitiva de la «quinta vía» como una analogía de proporcionalidad propia.

Por lo demás el paralelo no hace sino repetirse a lo largo del tratado, especialmente a partir del libro segundo, que versa «sobre la creación y las criaturas» (1261-1263), donde el capítulo primero comienza en exergo y deja expuesta la analogía de proporcionalidad de un modo preclaro con la cita del Salmo 145, versículo 5: «Meditatus sum in omnibus operibus tuis, et in factis manuum tuarum meditabar». En SCG lib. 2, cap. 37 matiza incluso cómo el nombre «producción» puede aplicarse al concepto de «creación» por una analogía de proporcionalidad propia, sin incurrir en contradicciones, ya que la potencia divina es infinitamente activa y sin privación. ${ }^{16}$

Según avanza el tratado Tomás afina con acuidad escolástica la argumentación conforme a estos tres ejes discursivos. Genera así formulaciones más completas y exhaustivas a partir de lo establecido en la primera. Este método

Journal of the History of Ideas, 1963, vol. 24, n ${ }^{\circ}$ 4, pp. 473-496..

12 Contra Gentiles, lib. 2 cap. 30 n. 14.

13 Contra Gentiles, lib. 2 cap. 46 n. 6.

14 Contra Gentiles, lib. 1 cap. 93 n. 4 .; lib. 2 cap. 26 n. 6.

15 Contra Gentiles, lib. 2 cap. 26 n. 9.

16 Contra Gentiles, lib. 2 cap. 37 n. 2; lib. 2 cap. 37 n. 4. 
de corrección progresiva se hace especialmente agudo en el libro tercero que acomete el tema de «Dios, fin último y gobernador supremo» (1263-1264). Las formulaciones de la «quinta vía» que desarrolla a partir de la primera exposición, explícita aunque promisoria, son las tres siguientes: A) la que depende de la unidad del orden cósmico garantizado por la unicidad de Dios: lib. 1 cap. 42 n. 7; B) la que procede del débito condicionado entre las diversas partes del orden cósmico: lib. 2 cap. $28 \mathrm{n}$. 15; y C) un simple epítome que resume lo ya enunciado en lib. 3, cap. 22, con ocasión del ordenamiento de los seres a su fin.

La primera formulación en Suma contra gentiles, lib. 1 cap. 13 n. 35, es bien conocida y reza como sigue: «Impossibile est aliqua contraria et dissonantia in unum ordinem concordare semper vel pluries, nisi alicuius gubernatione, ex qua omnibus et singulis tribuitur ut ad certum finem tendant. Sed in mundo videmus res diversarum naturarum in unum ordinem concordare, non ut raro et a casu, sed semper vel in maiori parte. Oportet ergo esse aliquem cuius providentia mundus gubernetur. Et hunc dicimus Deum».

El paralelo de este pasaje en Pugio fidei (I pars, caput II, ratio VII-VIII) reviste la forma convencional de una glosula al texto tomista, que repite el argumento del Damasceno. Las razones de Aristóteles no se aducen ni tampoco menciona el Comentario de Averroes, lo que demostraría una vez más la prioridad de Tomás como inspirador del tratado de Raimundo Martini, obra de categoría filosófica significativamente inferior. No obstante, el texto de Martini sirve para poner de relieve que el núcleo de la «quinta vía» en SCG es deudor sobre todo de los planteamientos de Metafísica XII 10 o Política I 8 pero no tanto Física II 8 como ocurre en la ST y que será el contexto determinante del argumento más acabado y definitivo.

Este pasaje en cuestión remite al argumento que San Juan Damasceno formula en los siguientes términos en Fides Orthodoxa: «Porro ipsa quoque rerum creatarum compages, conservatio, gubernatio, nos docet Deum esse, qui hoc universum coagmentavit et sustentat et conservat eique providet. Quomodo enim naturae inter se contrariae, ignis, inquam, et aquae, aeris et terrae, in unius mundi constitutionem convenissent, et indissolubili nexu cohaererent, nisi vis quaedam omnipotens ea compaginasset et perpetuo a dissolutione servaret? ${ }^{17}$

El texto de Averroes empleado como auctoritate resulta ser su comentario a la Física II, 8. Consideramos importante este detalle pues va a establecer la clave interpretativa justa para una cabal comprensión de la vía tal como quedará definitivamente fijada en ST, a través de los tramos argumentativos incoados en SCG: «quia ista propositio est máxima et fundamentum in hac scientia et in scientia divinia: quoniam, si naturalis non concesserit eam, negat principium finale et negat materiam esse propter formam, ex quo... et similiter si divinus non

17 De fide orthodoxa, I, c. 3 (PG. 94,795).

Contrastes vol. XXII-N² (2017) 
concesserit eam, non poterit probare quod Deus habet sollicitudinem circa ista quae sunt hic, ideo incoepit Aristoteles dicere quod natura agit propter alius». ${ }^{18}$

Comparando ahora entre sí los argumentos de ambas Sumas, se puede preguntar si se trata del mismo o de argumentos diferentes. Los mismos tomistas no están enteramente de acuerdo sobre esto. ${ }^{19}$ En SCG subordina la quinta vía a un corolario de la cuarta, operación que no se admite en la vía expuesta en ST. Por otra parte, en Contra gentiles parece referirse al orden finalístico del universo en su totalidad; mientras que en la Summa Theologiae toma como punto de partida de su prueba únicamente la finalidad como se manifiesta en los seres particulares de la naturaleza. ${ }^{20}$ Ademásen Contra Gentiles se comienza poniendo unas proposiciones o principios universales. En la Summa Theologiae se invierte este orden, para comenzar por los datos de experiencia. Sin embargo, no parece que éstas sean diferencias substanciales. Por lo que podría decirse que se trata del mismo argumento, bajo matices o versiones diferentes. ${ }^{21}$

Para establecer definitivamente los elementos que componen las vías sólo resta una última consideración sobre la ilación lógica que existe entre los términos que se expresan. En SCG encontramos las clasificaciones de una auténtica «filosofía de la técnica», en parte deudora de Aristóteles, pero con aportaciones originales, que se subordina al desarrollo de la analogía de proporcionalidad propia de la «vía teleológica».

Antes de exponer su transformación en ST debemos mostrar cómo estos análisis del fenómeno técnico, donde distingue los diversos tipos de artes y el orden jerárquico existente entre ellos, son parte esencial del curso evolutivo del argumento. De la simple lectura de estos textos fundamentales emanarán las claves para lo que consideramos el último y más profundo sentido de la «vía teleológica» como analogía de proporcionalidad propia.

Santo Tomás asume la distinción clásica aristotélica entre ciencias especulativas, ciencias prácticas y ciencias productivas, cada una de las cuales implica un arte diverso. ${ }^{22} \mathrm{El}$ arte práctico y el arte productivo se subordinan al arte

18 In II Physic., text. 75, t. 4 f.61v D (Venetiis apud Cominum de Tridino, Montis Ferrati, $1560)$.

19 Gilson, Etienne, «Trois leçons sur le problème de l'existence de Dieu», en: Divinitas, 1961 (5), pp. 61ss.

20 Kenny, Anthony, The Five Ways: St. Thomas Aquinas' Proofs of God's Existence, University of Notre Dame Press, 1980,pp. 93-131.

21 Cfr. Vicente Burgoa, Lorenzo, «Los problemas de la 'quinta via' para demonstrar la existencia de Dios», en Divus Thomas, 1981 (84), pp. 1-37. Kowalczyk, S. «L'argument de la finalité chez Thomas d'Aquin», en Divus Thomas, 1975 (78), pp. 47-48.

22 Aspe Armella, Virginia, El concepto de técnica, arte y producción en la filosofía de Aristóteles, FCE, México, 1993. Schummer, Joachim, «Aristotle on Technology and Nature», en: Philosophia Naturalis, $\mathrm{n}^{\circ}$ 38, 2001, pp. 105-120. De la Croce, Ernesto, «El concepto aristotélico 
especulativo, pero entre ambos encontramos una dependencia jerárquica donde el primero domina como superior al segundo. Cabe, por tanto, distinguir dos tipos de arte no especulativo: en primer lugar, un arte práctico cuyo fin es el perfeccionamiento de la acción misma, como el citarista ensaya para adquirir destreza en tañer la cítara; en segundo lugar, un arte productivo cuyo fin es generar algo exterior que la acción produce, tal como la casa es construida por el arquitecto.

A su vez distingue varias artes subsiguientes a cada una de estas: las artes prácticas incluirían las artes rectoras (prudencia política, mando militar, verbigracia) mientras que las artes productivas abarcarían las artes serviles (habilidad crematística, intendencia militar, pongamos por caso). Finalmente, y sin pretender ser exhaustivos, dentro de las artes productivas podemos distinguir entre el arte que impone formas a la materia como mera potencia pasiva (el cantero cuando talla la piedra inerte) y el arte que trabaja la materia por su potencia activa (el médico que cura el organismo aprovechando su poder regenerador).

Sirviéndose de esta clasificación general, Santo Tomás establece un orden jerárquico dentro de las ciencias y las artes. Las actividades productivas se subordinan a las actividades prácticas y ambas a la actividad especulativa. De manera que las ciencias prácticas son un analogado superior respecto de las actividades productivas. ${ }^{23}$ En consecuencia, las artes prácticas están ordenadas a las especulativas, porque toda operación humana se ordena a la especulación intelectual como a su fin. ${ }^{24}$ Pero el fin último de todas las ciencias y artes es propio de aquella a que se ordenan, la cual es directora y normativa de las demás; ${ }^{25}$ así el arte de navegar, al cual se ordena el fin de la nave, que es su propio uso, da normas y dirige al arte de construir naves. De manera que también hay una jerarquía entre las artes que fabrican el objeto y las artes que usan el objeto fabricado para su fin intrínseco. ${ }^{26}$ Hay un arte que opera con instrumentos para producir la obra y hay un arte sin instrumentos que usa la obra producida como medio para un fin..$^{27}$ Aquellas especies de arte se subordinan a estas otras especies artísticas. ${ }^{28}$

de técnica», en: Ethos. Revista de Filosofía Práctica, no 4 y 5, 1979, pp. 253-264.

23 Contra Gentiles, lib. 3 cap. 76 n. 4-7.

24 Contra Gentiles, lib. 3 cap. 132 n. 13. Quaestio de opere manuali. De opere manuali religiosorum (1255-1256), en: Quodl. VII, q 7. Suma Theologiae, II'-IIae, q. 187 a. 3 arg. 4.

25 Contra Gentiles, lib. 2 cap. 42 n. 5

26 Contra Gentiles, lib. 2 cap. 92 n. 6.

27 Contra Gentiles, lib. 2 cap. 92 n. 3: «Sicut, si instrumenta artificialia sunt propter homines qui per ea operantur, nihil prohibet esse alios homines qui per instrumenta huiusmodi non operantur immediate, sed imperant operantibus».

28 Contra Gentiles, lib. 3 cap. 25 n. 9. 
El primer tipo de artista produce el artefacto confiriéndole una forma capaz de alcanzar su fin específico; el segundo tipo de artista se sirve del artefacto para alcanzar en acto dicho fin. ${ }^{29}$ Hay, por tanto, una relación de potencia y acto del artefacto respecto de su finalidad: el primer arte trabaja ciertos materiales para obtener artefactos, el segundo arte ordena el artefacto hacia su finalidad intrínseca. ${ }^{30} \mathrm{La}$ analogía de proporcionalidad establece que Dios, como análogo propio, es productor de la virtualidad de los seres naturales, como el primer género de artífice, ${ }^{31}$ pero también conserva en el ser la actualidad teleológica de los seres, como el segundo género de hombre técnico, a la manera del fabricante de flechas y el lanzador de flechas respectivamente. ${ }^{32}$ La técnica de fabricar flechas se subordina entonces a la técnica de lanzarlas hacia un blanco, porque el arte que mira al fin es más perfecto que el arte que mira al objeto. ${ }^{33}$ No obstante, decimos que la providencia de Dios es un arte según las dos formas. ${ }^{34}$

El analogado principal de esta analogía de atribución entre las diversas artes mencionadas corresponde al «sagitante» como «lanzador de flechas». ${ }^{35}$ Sin embargo, el fin del lanzador de flechas y el fin del fabricante de flechas es el mismo e inmanente a la flecha; este fin es objetivo e intrínseco al dardo cuando se identifica con el blanco o la diana, de manera que el finis operantis de ambos «flechadores» queda objetivado en el finis operis de la «flecha». ${ }^{36}$ Insistimos en este ejemplo analógico de Tomás porque va a estar contenido en todos los formatos de la «quinta vía» como esquema fundamental hasta desembocar finalmente en la ST.

\section{La «Quinta VÍA» EN la Suma Teológica.}

Aparte de la exposición escolar ${ }^{37}$ explícita de la quinta vía tomista de la ST (I, q. 2, art. 3), encontramos otras implícitas. ${ }^{38} \mathrm{En}$ ST asume de raíz la estructura lógica del argumento como analogía de proporcionalidad propia y la expone de un modo más claro que en SCG. La vía intenta conciliar las consecuencias que impone racionalmente la reducción dialéctica perpetrada por Aristóteles en Física II 8 con el dogma cristiano de un Dios creador y providente.

29 Contra Gentiles, lib. 3 cap. 111 n. 1.

30 Contra Gentiles, lib. 4 cap. 75 n. 1.

31 Contra Gentiles, lib. 4 cap. 35 n. 4.

32 Contra Gentiles, lib. 3 cap. 64 n. 2

33 Contra Gentiles, lib. 3 cap. 24 n. 4 y 5 .

34 Contra Gentiles, lib. 3 cap. 64 n. 5; lib. 3 cap. 80 n. 4

35 Contra Gentiles, lib. 3 cap. 78 n. 4

36 Contra Gentiles, lib. 3 cap. 2 n. 1-2.

37 S. Th. I q. 2 a. 3 co.

38 S. Th. I $I^{\mathrm{a}}$ q. 103 a. 1 ad 1. 
Como es bien sabido, Aristóteles en Física II 8 establece una reducción dialéctica sobre la teleología de los seres biológicos. ${ }^{39}$ Dicha reducción pretende establecer el modo de pensar la finalidad de modo apodíctico. ${ }^{40} \mathrm{Como}$ tal, la reducción formularía el modo necesario de pensar la finalidad ${ }^{41}$ tanto para afirmarla como para negarla, radicalizarla o moderarla, puesto que no se puede pensar sobre la finalidad si no es conforme a lo concluido en la reducción. ${ }^{42}$

Entre otras consecuencias la reducción establece que el azar (tyché) deriva de la materia de los seres finalizados y que, por tanto, el azar no es una entidad subsistente que exista al margen de los seres naturales. ${ }^{43}$ De lo contrario el azar quedaría hipostasiado, sustantivado, como una personificación o una figura mítica, y dejaría de ser un simple nombre lógico que, como etiqueta, designa la concurrencia de causas impropias que perturba la cadena generativa de los animales. Por tanto, la finalidad introduce la necesidad y la materia, la excepción accidental.

Aristóteles recorta, de esta manera, el azar y la necesidad dentro del proceso inmanente de generación y corrupción de los individuos finalizados. Cuanto queda fuera de los individuos teleológicamente conformados queda vaciado, anulado racionalmente, y no puede ser pensado como «origen de las especies».

39 Ariew, André, «Platonic and Aristotelian Roots of Teleological Argument», en: Functions. New Essays in the Philosophy of Psychology and Biology, Ariew, Cummins y Perlman (ed.), Oxford, Oxford University Press, 2002, pp. 7-32. Mansion, A., Introduction à la physique aristotelicienne, Lovaina, Éditions de l'Institut Supérieur de Philosophie, pp. 80ss. Jaume, Andrés L., «La teleología aristotélica como una inferencia a la mejor explicación: un análisis epistemológico del principio de finalidad en el libro II de la Física de Aristóteles», Agora: papeles de Filosofía, 2013, 32/2, pp. 29-47.

40 Nagel, Ernst, La estructura de la ciencia, Paidós, Barcelona 2006, pp. 256-257. La teoría biológica presenta en su contexto de justificación -y no ya solo en su contexto de descubrimiento- una acumulación evidente de léxico formalmente teleológico sin el que la biología no podría conceptualizarse ni existir. La reducción de Física II 8 establece los modi sciendi gnoseológicos de la biología, los cuales interponen las relaciones necesarias entre los términos del campo biológico, de suerte que ellos mismos no podrán en modo alguno quedar eliminados, sin que el propio tejido categorial de la biología sufra una enérgica distorsión en muchos de sus sectores, lo mismo a efectos sintácticos, como semánticos o pragmáticos.

41 «Pues también esto último [eneká, el fin, «aquello para lo cual»] es principio no de la acción sino del raciocinio (y también allí [en las matemáticas] lo es del raciocinio, pues no hay acciones)». Física II 9, 200 a 23-24. Cfr. De part. Anim., A, I, 639 b 12-640 a 1-9.

42 Düring apunta que, a menudo, cuando Aristóteles habla de causalidad confunde la ratio essendi con la ratio cognoscendi. Dar razón de algo no es lo mismo que decir que algo sea el caso. Sin embargo, que la reducción pertenezca al orden del conocimiento no menoscaba sus conclusiones, ya que es imposible pensar de otra manera los procesos materiales donde que se dan necesariamente. Cfr. Düring, Ingemar, Aristóteles, México, FCE, 2005, pp. 321ss.

43 Gabriela, Rossi, El azar según Aristóteles. Estructuras de la causalidad accidental en los procesos naturales y en la acción, Academia Verlag, 2011. 
De manera que no sólo el azar sino también la causa eficiente queda, en consecuencia, neutralizada: no puede admitirse en el exterior de la inmanencia teleológica una causa eficiente de la finalidad que confiera las formas a los individuos. ${ }^{44}$

Santo Tomás asume esta reducción aristotélica de Física II 8, que hace imposible pensar una causa eficiente de los seres naturales finalizados. Pero consigue conciliar esta conclusión con el dogma cristiano de un Dios creador del mundo. Para compatibilizar ambas posturas, aparentemente irreconciliables, procede en dos momentos bien diferenciados:

A ) En un primer momento expone cómo se obtiene la idea de Dios como «inteligencia ordenadora» desde los seres finalizados a través de una analogía de proporcionalidad propia. De esta manera, la idea de Dios como «inteligencia ordenadora» no vulnera la inmanencia incausada de las especies naturales y sus dinamismos teleológicos autosuficientes, según la reducción aristotélica.

B) En un segundo momento introduce esta idea como el analogado supremo de una analogía de atribución a través de la doctrina metafísica de la participación. De esta manera, conecta de nuevo este orden cósmico de especies finalizadas con las exigencias doctrinales de un Dios creador y providente, y evita convertir la analogía de proporcionalidad inicial en una simple metáfora.

La exposición más clara de la mencionada analogía de proporcionalidad será el escolio a la vía apuntado en Ia-IIae q. 13 a. 2 ad 3. Se conserva incluso el paralelo establecido en SCG entre los diversos tipos de artes, prácticas y productivas, en el seno de la analogía. ${ }^{45}$ De esta manera, la analogía de proporcionalidad propia surge como corolario derivado de la reducción dialéctica formulada por Aristóteles en Física II $8 .{ }^{46}$

El enunciado «Dios existe» se manifiesta como verdadero en la «quinta vía» a través de una analogía compuesta de cuatro términos que se relacionan en paralelo duorum ad duo, según el cuarto tipo irreductible de relación análoga. ${ }^{47}$

44 Metafísica VII 1032 a 12-1034 b 19; XII 1, 1069 b 6. Física I 6-10. Generación y Corrupción XI 337 b 34-338 b 25.

45 S. Th. I q. 19 a. 4 co.

46 Comentario a la Física de Aristóteles, libro II, capítulo 8, lectio 14.

47 Las evidencias textuales de esta analogía de proporcionalidad propia en la Prima pars de la Summa Theologiae son innumerables. Cfr. ST I ${ }^{\mathrm{a}}$ q.8 a. 4; $\mathrm{I}^{\mathrm{a}}$ q.9 a.1 resp. 2; $\mathrm{I}^{\mathrm{a}}$ q.14 a.8; $\mathrm{I}^{\mathrm{a}}$ q.14 a.12; I $I^{\mathrm{a}}$ q.17 a.1; $\mathrm{I}^{\mathrm{a}}$ q. 21 a.2-3, sol.; $\mathrm{I}^{\mathrm{a}}$ q. 22 a.2-a.3; $\mathrm{I}^{\mathrm{a}}$ q. 23 a.6; $\mathrm{I}^{\mathrm{a}}$ q. 27 a.2 $; \mathrm{I}^{\mathrm{a}}$ q. 44 a.3; $\mathrm{I}^{\mathrm{a}}$ q.45 a.6; I ${ }^{\mathrm{a}}$ q.63 a.1; $\mathrm{I}^{\mathrm{a}}$ q.65 a.3 resp. $2 ; \mathrm{I}^{\mathrm{a}}$ q.65 a.4; $\mathrm{I}^{\mathrm{a}}$ q.77 a.3; $\mathrm{I}^{\mathrm{a}}$ q.91 a.3 sol. 
De este orden son las proporciones matemáticas de Eudoxo ${ }^{48}$ (1/3 es a 2/4 lo que 2/6 es a 4/8) o las comparaciones ejemplares de Aristóteles en la Poética: «El escudo es a Ares lo que la copa es a Dioniso» ${ }^{49}$ que son las formas más primitivas de este tipo analógico.

La analogía de proporcionalidad propia de cuatro términos relacionados dos a dos queda establecida como sigue: «como las cosas artificiales se comparan al arte humano, así las cosas naturales al arte divino». ${ }^{50}$ La quinta vía tal como se formula en ST compendia la evolución del argumento en SCG, y lo enuncia de un modo icástico. En ambas Sumas extrae posteriormente todas las consecuencias de este concepto prolongando el uso de la analogía a las coincidencias y las diferencias entre los seres naturales y artificiales, mostrando asî que se establece una analogía de propiedades esenciales y no una comparación aparente o metafórica. ${ }^{51}$

Esta sencilla analogía de proporcionalidad acuñada canónicamente en SCG y asumida por entero posteriormente en ST no es, sin embargo, una proporción cuantitativa sino de propiedades metafísicas. Tampoco es una mera metáfora o analogía de proporcionalidad impropia, sino que establece una proporcionalidad propia y esencial de distintos términos. Pensamos en Dios como «gobernador del cosmos» cuando hemos aplicado la analogía de proporcionalidad entre los artefactos técnicos y los seres naturales del universo considerado como un todo.

Sostenemos que la «quinta vía» es esencialmente la misma para ambos tratados y que en su estructura lógica. En la fórmula de ST Tomás omite la analogía de atribución precisamente porque asume las consecuencias de la reducción dialéctica de Física II 8, según el cual no es posible pensar una causa productora de la finalidad. Es decir, no se puede pensar en Dios respecto del orden teleológico con una analogía de atribución, como si Dios fuera la causa agente de la teleología biológica. Pero muestra cómo la idea de Dios como gobernador del mundo se impone por analogía de proporcionalidad a partir de la reducción dialéctica operada por Aristóteles en Física II 8.

En SCG la prioridad de la obra es la polémica contra los adversarios dialécticos y sus discursos contra la providencia, o clarificadora de los discursos favorables pero erróneos que requieren ser corregidos y mejorados. Esta idea de Dios como «inteligencia ordenadora» sirve para purificar desde la teología preambular los discursos y auctoritates acerca de la providencia divina formulados

48 Ross, David, Aristóteles, Ed. Sudamericana, Buenos Aires, 1957, pp. 54-55.

49 Poética, 21, 1457 b, 16ss. Pierre Aubenque, Le prohleme de l'etre chez Aristote, P.U.F., París, 1972, p. 202.

50 Establece la analogía incluso entre el arte humano y el arte trinitario: Ia q.36 a.3; q.36 a.4; q.39 a.8. «Esto mismo lo sugiere Agustín cuando dice: Como palabra perfecta a la que no le falta nada, especie de arte del Dios omnipotente». I' q.39 a.8 con. 4; q.74 a.3 resp.

51 S. Th. I' q. 103 a. 1 ad 3. 
por los artistas, comentaristas y teólogos, sean cristianos o no. Con este criterio de juicio somete a crítica los argumentos referidos a la providencia divina (lib. 3, caps. 64-76) y el gobierno divino (lib. 3, caps. 77-110), para proceder a una «reducción dialéctica» contra los que niegan la finalidad y la creación. Obtiene también así Tomás una «corrección dialéctica» para los argumentos que afirman, pero de manera equivocada, sobre el mismo asunto.

En ST podemos presentir, más bien, que Tomás estaba buscando la mejor manera de redactar una exposición sintética y de conjunto de la Teología, donde deseaba subrayar la doctrina de la creación. La idea de Dios como «inteligencia ordenadora» se integra en una doctrina de la creatio ex nihilo donde se conjuga la participación platónica con el causalismo aristotélico de un modo singular, ya que la causalidad divina no se entiende como «eficiencia extrínseca» sino como emanación participativa desde el máximo. ${ }^{52}$ Distribuye el tema de la procesión de todas las criaturas a partir de Dios en tres grandes secciones: la producción de las criaturas (qq. 44-46), su distinción (qq. 47-102), y su conservación y gobierno (qq. 103-119). Tomás no incumple las conclusiones necesarias de Física II 8 porque concibe la causalidad como participación metafísica desde la actividad divina y no como una causalidad agente extrínseca.

\section{CONCLUSIÓN.}

Es bien conocida la disputa sobre la evolución del pensamiento tomista con respecto a la doctrina de la analogía. ${ }^{53}$ No es este el momento adecuado para entrar a tomar partido por ninguna de las posiciones principales de la polémica, pero debemos mencionar tangencialmente la cuestión en la medida en que afecta a nuestro tema de análisis. Según hemos expuesto quedaría en evidencia que tanto en la SCG como en la ST la quinta vía acusa la estructura lógica de una analogía de proporcionalidad propia, pero cada Suma aplica los

52 González, Ángel Luis, Ser y participación: estudio sobre la cuarta vía de Tomás de Aquino, EUNSA, Navarra, 1979, pp. 173-245. Artola, José María, Creación y participación, Madrid, 1963, pp. 83ss. Fabro, Cornelio, Partecipazione e causalità secondo S. Tommaso d'Aquino, Torino, 1960. Ibídem, La nozione metafísica di partecipazione secondo S. Tommaso d'Aquino, Torino, 1963. Geiger, L. B., La participation dans la philosophie de S. Thomas d'Aquino, París, 1953.

53 Entre los autores que sostienen que la posición del Aquinate sobre el primado metafísico de una u otra analogía sufre variaciones a lo largo de sus escritos: Bastit, Michel, Les principes des choses en ontologie médiévale, Bordeaux, éd. Bière, 1997, pp. 60ss. Klubertanz, George P., St. Thomas Aquinas on Analogy: A Textual Analysis and Systematic Synthesis, Chicago, Loyola University Press, 1960. Montagnes, Bernard, La Doctrine de l'Analogie de L'Être d'après Saint Thomas d'Aquin, Louvain, Paris, 1963. McInerny, Ralph, Aquinas and Analogy, Washington, The Catholic University of America Press, 1996. 
réditos de esta proporcionalidad desde un procedimiento argumentativo fundamentalmente diverso.

Como queda dicho, en SCG prevalece un uso lógico con fundamento in re de la analogía, de manera que el término proporcionado del «artesano divino» sirve como criterio de juicio para una «reducción dialéctica» de la providencia y el gobierno divino, frente a las éndoxa de los discursos partidarios deficientes o los argumentos adversos.$^{54}$ Hay autores que consideran que este punto de vista lógico de la analogía es el fundamental y primario en Tomás. ${ }^{55}$

Sin embargo, en ST domina más bien un uso metafísico de la analogía donde la lógica de las proporciones queda integrada bajo la doctrina de la participación platónica, de manera que la «inteligencia ordenadora» por eminencia se manifestaría como origen de un mundo jerárquico de seres ordenados en grados de mayor a menor perfección. ${ }^{56}$ Esta perspectiva es reivindicada por los tratadistas que subordinan la predicación análoga a su fundamento. ${ }^{57}$

Dicho planteamiento resulta coherente con el carácter polémico de SCG, pese a su exposición doctrinal; y, paradójicamente, este tratamiento de la analogía conviene a la finalidad doctrinal de un texto tan dialéctico como la ST. Sin embargo, ambos usos no son mutuamente excluyentes y encontraremos inserciones abundantes de uno y otro modo en el tema dominante de las respectivas Sumas.

Afirmamos que el predominio de un planteamiento sobre el otro no supone la exclusividad absoluta de un único procedimiento. La razón de esto obedece a que en la concepción tomista de la analogía se entrecruzan sin duda varias corrientes filosóficas. En este caso, no sólo la idea de «participación» que asume de Platón (comprensión de la realidad en términos de modelo eminente e imagen imperfecta y, por tanto, su distinción y coincidencia) sino, sobre todo, la «unidad de relación de semejanza» o relación objetiva (proporcionalidad) que toma de Aristóteles. Finalmente, la «unidad de referencia a un primero» («per prius et posterius»), o atribución, que toma del mismo Aristóteles y de la filosofía judeo-árabe, y que implica la idea de un mundo ordenado por relaciones

54 Bellerate, B. M., L'analogia tomista nei grandi commentatori di S. Tommaso, UPS, Roma, 1960, cap. I y II.

55 McInerny, Ralph, The Logic of Analogy: An Interpretation of St. Thomas, The Hague, Martinus Nijhoff, 1961, pp. 30ss. Manser, G., La esencia del tomismo, Madrid, CSIC, 1947, pp. 500-528.

56 Lyttkens, Hampus, The Analogy Between God and the World: An Investigation of its Background and Interpretation of its use by Thomas of Aquino, Uppsala, 1952. Montagnes, Bernard, La Doctrine de l'Analogie de L'Être d'après Saint Thomas d'Aquin, Louvain, Publications Universitaires, 1963, pp. 156-166.

57 Anderson, James F., Reflections on the Analogy of Being, The Hague, Martinus Nijhoff, 1967. 
de causalidad, ${ }^{58}$ le permite evitar el riesgo de la equivocidad. La tesis que, sin embargo, no aceptamos en ningún caso es la que postula reducir la analogía de proporcionalidad propia empleada en ambos tratados a una analogía de atribución encubierta, reducción que obedeciera a una supuesta evolución del pensamiento del Doctor Angélico. ${ }^{59}$

El argumento, en uno y otro caso, proporciona a Dios el nombre de «inteligencia ordenadora» de forma conceptual, no tanto ordo essendi como ordo cognoscendi, aunque esta relación de términos sea necesaria e impensable de otro modo. Es cierto que, posteriormente, en lo que respecta a la aplicación de esta noción a la providencia y el gobierno divino emplea, de manera central, la analogía de proportio o atribución, para subrayar la dependencia de las criaturas con respecto al Creador y evitar así la equivocidad. ${ }^{60}$ Pero la analogía de proporcionalidad no se emplea de manera secundaria y derivada solamente para remarcar la distancia infinita entre criatura y Creador, sino que es el núcleo esencial del argumento mismo referido a los seres naturales finalizados, de donde obtenemos la noción misma del Creador como «artesano divino» y de la creación como «producción divina». El uso hegemónico que se haga de este concepto límite será lo que distinga a una Suma de la otra. ${ }^{61}$

58 Schillebeeckx, E. Revelación y Teología, Salamanca, Sígueme, 1968, pp. 295-321.

59 Hay autores que resuelven esta paradoja con la tesis inversa: no se trata un uso diverso de la analogía de proporcionalidad en una y otra obra sino un uso diverso de la participación entre ambos períodos. Cfr. Geiger, L. B., La participation dans la philosophie de B. Thomas d'Aquin, J. Vrin, 1953, pp.157-244. La tesis de Geiger afirma que hay dos concepciones de la participación, previas al Doctor Angélico, que él unificará posteriormente. Una primera idea de la participación procede del aristotelismo y recibe el nombre de participación por composición. La variedad de los seres, donde se mezcla la unidad y la diversidad, se explica por medio de la composición de la forma inherente. La segunda concepción de la participación considera que las esencias se escalonan en modos y grados que, por perfecciones deficientes, introducen la limitación respecto a la perfección suma.

60 ST, I q.4 a.3; I q.6 a.4; I C.13 a.7.

61 En este sentido asumimos y matizamos la interpretación que sostiene Enrico Berti sobre el tipo de analogía dominante en ambas Sumas. Enrico Berti considera que en las obras juveniles (Los principios de la naturaleza y el Comentario a las Sentencias) santo Tomás sostiene la primacía de la analogía de atribución, entendida como expresión de una gradación existente entre los entes, es decir, de una perfección común poseída en grados diversos. En cambio, en Sobre la verdad, atribuye el primado a la analogía de proporcionalidad por el descubrimiento de la tesis aristotélica según la cual «el infinito no está en alguna relación con lo finito». En las dos Sumas y en el Comentario a la Metafísica regresa al primado de la analogía de atribución, y ello porque en estas últimas obras no se entiende el ser como forma sino como acto, y la causalidad divina ya no es vista como causalidad ejemplar o formal sino esencialmente como causalidad eficiente. Cfr. Berti, Enrico, «L'analogia dell'essere nella tradizione aristotelico-tomista», en: VVAA, Metafora dell'Invisibile. Contributi al XXXVIII Convegno del Centro di Studi filosofici di Gallarate, Morcelliana, Brescia 1994, pp. 13-33. 
La reducción dialéctica de Física II 8 sobre la teleología es la razón necesaria y suficiente que explica este punto de partida único. La constitución lógica de la «quinta vía» en SCG y su posterior transformación en ST, tal como queda apuntado en este artículo, son las razones que ofrecemos para rechazar las interpretaciones contrarias a nuestro planteamiento.

Desiderio Parrilla Martínez es doctor en Filosofía por la Universidad Complutense de Madrid y profesor adjunto del departamento de humanidades en la Universidad Católica de Murcia (UCAM).

Líneas de Investigación:

Vicepresidente de la asociación de estudios girardianos Xiphias Gladius (www.violenciayreligion.org), asociada al grupo internacional de investigación Imitatio (http://www.imitatio. org/) y la Peter Thiel Foundation.

Publicaciones recientes:

«¿Hay un "perdón al enemigo" en el estoicismo antiguo?» Anales del seminario de historia de la filosofía, Vol. 33, Vol. 2, 2016, pp. 419-444.

«Los límites de la teoría mimética como categoría política», en: David Atienza de Frutos y David García-Ramos Gallego (eds.), La construcción de la identidad en tiempos de crisis. El papel de la violencia y la religión, ISBN: 978-84-16421-62-6, Barcelona, Ed. Anthropos, 2017, pp. 81-107.

«Mimesis, Ritual Sacrifice, and Ceremony of Proskynesis», Contagion: Journal of Violence, Mimesis, and Culture, Vol. 24, No. 1 (Spring 2017), pp. 57-72.

René Girard: la violencia desvelada, ISBN: 9788491482857, Madrid, Dykinson, 2017.

Correo electrónico: dparrilla@ucam.edu 